\title{
ASSESSMENT OF ECONOMIC CONSIDERATIONS FOR AIR POLLUTION CONTROL TECHNOLOGIES
}

\author{
MORIDI, F. ${ }^{1}-$ YARAHMADI, R. $.^{2 *}-$ ABDOLI-SENJANI, M. ${ }^{1}-$ MARJANI, A. ${ }^{1}$ \\ ${ }^{I}$ Department of Chemistry, Arak Branch, Islamic Azad University, Arak, Iran \\ ${ }^{2}$ Air Pollution Research Center, school of public health, Iran University of Medical Sciences, \\ Tehran, Iran \\ *Corresponding author \\ e-mail: yarahmadi.r@iums.ac.ir; phone: +98-21-8889-7613 \\ (Received $9^{\text {th }}$ Jun 2018; accepted $14^{\text {th }}$ Aug 2018)
}

\begin{abstract}
Today, advances in technology help humans to overcome emissions to some extent. Environmental researchers have attempted in recent years to find low-cost and effective approaches to pollutants control. It is important to consider the economic sphere in the approaches of dealing with pollution, as it has merits that should not be ignored, because of the existence of inefficient and expensive rules. To control air pollution, effective and long-term strategies should be identified and implemented. Among the components of air pollution control systems, pollution remediation equipment is of high importance. The present study aimed to assess air filtration systems, with emphasis on hybrid and new technologies, economic evaluation and prioritization of particle control technologies using a cost-benefit analysis method. According to the results, the research Cost-benefit analysis of particles control technologies based on research aspect had different weights. Wet ESP, Nano fiber beds, and ESP Bag Filter respectively were identified as the most appropriate particulates control techniques.
\end{abstract}

Keywords: particle filtration, priority, equipment, new technology, air pollution

\section{Introduction}

Industrial and technological developments and increasing energy consumption have led to renewable sources pollution (Zhang et al., 2015; Zeng et al., 2017). Given operating costs of different methods of pollution control and lack of return on investment have become air pollution control into an unprofitable economic burden (Robert and Erson, 1983; Moridi et al., 2017; Yarahmadi et al., 2010). To control air pollution, effective and long-term strategies should be identified and implemented (Vlachokostas et al., 2011; Yarahmadi et al., 2010; Lei, 2005; Vanoye and Mendoza, 2014; Chen et al., 2018). However, most decision-makers are not able to properly assess and reduce the costs of environmental degradation and the expenditure necessary to comply with the standard rules of air pollution reduction. For this reason, in many cases managers have had to use methods that are either expensive and unaffordable, or inexpensive but unreliable. Engineering Economics is a specialized field in economics that uses analysis methods to estimate costs (Zhao et al., 2016) and determine the value of industrial projects. This field has many roles, including the evaluation of industrial project usefulness, estimating values, and evaluating them in terms of technical and engineering issues (Salehi and Tavakkoli-Moghaddam, 2008). General methods used to particles are settling chamber, cyclone, bag house, scrubber, ESP and extra. The final composition is applying control measures and using technical equipment based on economic aspects (Boardman et al., 2017; Jin et al., 2018; Min et al., 2017). The absence of relevant studies can lead to health, safety, economic, energy, environmental $\left(\mathrm{HSE}_{3}\right)$ and social losses; this research was the first to prioritize and select the most 
appropriate particles control hybrid and new technologies based on cost benefit analysis. Effective and affordable control measures are an optimal solution for administrative-managerial considerations (Murty et al., 2006; Nath and Cholakov, 2009; Yarahmadi and Sadoughi, 2012; Spiegel and Maystre, 1998). Previous studies indicate that, the high initial and operative costs of common filtration methods and lack of return on investment have turned air pollution control into an unprofitable economic burden. Based on these concern policy makers have hence made numerous efforts to develop cost-effective methods and optimized techniques to manage air pollution based on all economic, environmental, and social considerations. Therefore, selection of air pollution new technologies have been proposed to prioritize and chosen by seeking an option which provides maximum benefit based on all mention criteria (Moridi et al., 2017).

These also include the cost of raw materials, design costs, consulting costs, personnel costs and maintenance costs. A study entitled cost analysis framework project was published; it used cost-benefit analysis as a practical tool for evaluating different types of projects. This study aimed to analyze costs and benefits, assess money value, and offer the highest quality of results in order to optimize the use of valuable resources (Qld, 2011). The most common method for assessing the beneficial effects of environmental policies is cost-benefit analysis (CBA) (Voorhees et al., 2001; Gao et al., 2016; Bollen et al., 2009; Huang et al., 2017; Sen, 2000).

Robert et al. (1983) considered the economic sphere in the approaches of dealing with pollution to have merits that should not be ignored, as inefficient and expensive rules exist (Robert and Erson, 1983). Studies show that in most cases, the most costeffective pollution control program is the cheapest one (Yarahmadi et al., 2012). The aim of research is assessment of new technologies with economic, environmental, social consideration for particulate control technologies using a cost-benefit method.

\section{Methods}

The cost-benefit analysis (CBA) method is often used as an efficient tool for estimating environmental costs and benefits. This method is used in decision-making relating to development projects as well as the evaluation of policies (Voorhees et al., 2001; Quah, 2007).

This method, which uses the simple cost-benefit approach tool (SCBAT), selects the best option in regard to economic-executive considerations that help project managers in managerial decision-making. This method was used for optimized air filtration technologies selection with the cost-benefit aspects include of Financial, Personnel, Service and maintenance, Environmental-social, Production and HSE risks for the first. Using this method in our study, the procedure is as follow (Cellini and Kee, 2010):

1. A list of innovative technologies in the field of particles control was collected.

2. Experts assessed the initial survey list. Determination the aspects of costbenefits associated for key selected technologies were done by expert panel groups (brainstorm).

3. Criteria for the costs and benefits and their elements associated with each option of the investigated technologies in Table 1 were identified (Treasury, 2006; Campbell and Brown, 2003). 
4. According to the method, the criteria of cost benefit were scored through linguistic variables (scores: $1-3$ Scale Rating: Low $=1$, Moderate $=2$, High $=$ $3)$.

5. The benefit-to-cost ratings ratio and the ranking by the outcome of technology from benefit-cost standards were determined (Svensson, 2016).

6. If the resulting number is greater than one, it means there is more profit than cost and high priority will be given. If the number is one, the benefit is equal to the cost, and priority will be given with caution. If the benefit ratio is less than the cost and the obtained value is smaller than one, the action will be in completion. Cost-benefit in this case does not have any benefit, and this option will not be given priority.

7. Setting priorities based on larger concessions will be a top consideration.

According to the method, a list of hybrid and new control equipment for particle air pollutants was given to the experts to be indexed. In this study, an expert panel of six technicians with high experience in the fields of air pollution, environmental sciences, occupational health and industrial safety was used. The present study expert panel of was selected of four company in Iran with the ability of design, manufacture, install, and maintenance. The criteria of each cost-benefit exercise and their constituent elements associated with each option were scored by experts and specialists from design and manufacturing companies. Then, the benefit-to-cost ratio was calculated and ranked.

Table 1. Aspects of costs and benefits associated with each action

\begin{tabular}{c|c}
\hline The cost-benefit aspects & More description \\
\hline Financial & Investment - required equipments to build and launch \\
Personnel & Training - internships - internal, external period - absence from work \\
Service and maintenance & $\begin{array}{c}\text { Operating period - services - operational energy - maintenance and service } \\
\text { Environmental-social } \\
\text { Release of toxic substances - waste management - the rule of law - efficiency } \\
\text { Customer orientation } \\
\text { Production }\end{array}$ \\
HSE risks & $\begin{array}{c}\text { Investment - new products - the need of new information } \\
\text { Development - efficiency - replacing equipment and entrance fees }\end{array}$ \\
\hline
\end{tabular}

\section{Results}

Initial and selected list of particulate control technologies were shown in Table 2. Information collected in the Tables 3-7 for rating particle-filtering technologies after the preliminary analysis:

Table 2. Initial and selected final particulate air pollution control

\begin{tabular}{c|c|c|c}
\hline \multicolumn{3}{|c|}{ Initial list } & Key selected technologies by expert panel \\
\hline Bag house & Wet ESP & Fabric filters & ULPA Filter \\
Settling chamber & Nano fibers bed & HEPA Filter & Wet ESP \\
ULPA filter & Scrubber & Wet cyclone & Nano fibers bed \\
Cyclone & ESP bag filter & Multicyclone & ESP Bag filter \\
\hline
\end{tabular}


Table 3. Results of cost-benefit criteria and elements evaluation for ULPA Filter

\begin{tabular}{|c|c|c|c|c|c|}
\hline $\begin{array}{l}\text { Cost-Benefit } \\
\text { criteria }\end{array}$ & $\begin{array}{l}\text { Elements of cost- } \\
\text { benefit criteria }\end{array}$ & $\begin{array}{l}\text { Benefit elements } \\
\text { examples }\end{array}$ & $\begin{array}{l}\text { Average } \\
\text { benefit } \\
\text { rate }\end{array}$ & $\begin{array}{l}\text { Examples of cost } \\
\text { elements }\end{array}$ & $\begin{array}{l}\text { Average } \\
\text { cost rate }\end{array}$ \\
\hline Financial & $\begin{array}{l}\text { Investment, } \\
\text { required } \\
\text { equipments to } \\
\text { build and launch }\end{array}$ & 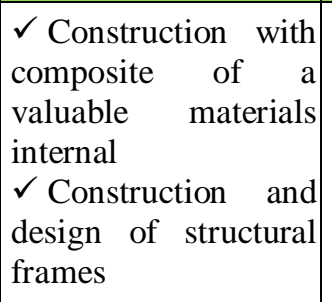 & 1.66 & $\begin{array}{l}\checkmark \text { Assembled and the } \\
\text { possibility changes in } \\
\text { the original design } \\
\checkmark \text { The need for special } \\
\text { compounds against heat } \\
\text { high }\end{array}$ & 2.33 \\
\hline Personnel & $\begin{array}{l}\text { Training, } \\
\text { internships, } \\
\text { internal, external } \\
\text { period, absence } \\
\text { from work }\end{array}$ & $\begin{array}{l}\checkmark \text { Need to launch } \\
\text { scientific education } \\
\text { course }\end{array}$ & 1.66 & $\begin{array}{l}\checkmark \text { Training of } \\
\text { technicians for technical } \\
\text { changes in the structure }\end{array}$ & 2.33 \\
\hline $\begin{array}{l}\text { Service and } \\
\text { maintenance }\end{array}$ & $\begin{array}{l}\text { operating period, } \\
\text { Services, } \\
\text { Operational } \\
\text { Energy, } \\
\text { Maintenance } \\
\text { service }\end{array}$ & $\begin{array}{l}\checkmark \text { Reuse the filter } \\
\text { after cleaning } \\
\checkmark \text { Very low energy } \\
\text { consumption }\end{array}$ & 2 & $\begin{array}{l}\checkmark \text { High level } \\
\text { maintenance service } \\
\checkmark \text { Blocked pores } \\
\text { through time } \\
\checkmark \text { Damage Due to heat }\end{array}$ & 1.66 \\
\hline $\begin{array}{l}\text { Environmental } \\
\text {-social }\end{array}$ & $\begin{array}{l}\text { Release of toxic } \\
\text { substances, Waste } \\
\text { Management, the } \\
\text { rule of law, } \\
\text { efficiency }\end{array}$ & $\begin{array}{l}\checkmark \text { High purification } \\
\text { efficiency }\end{array}$ & 2.33 & $\begin{array}{l}\checkmark \text { toxic gas emissions } \\
\checkmark \text { Blocked pores } \\
\text { through time and } \\
\text { quality loss }\end{array}$ & 1.33 \\
\hline $\begin{array}{l}\text { Customer } \\
\text { orientation }\end{array}$ & \begin{tabular}{|lr} 
Investment, new \\
products, the need \\
of \\
information
\end{tabular} & $\begin{array}{l}\checkmark \text { This technology is } \\
\text { native } \\
\checkmark \text { In plaster and } \\
\text { cement factories are } \\
\text { part of a high quality } \\
\text { product }\end{array}$ & 1.33 & $\begin{array}{l}\checkmark \text { Require a regenerator } \\
\text { needed for recycling } \\
\checkmark \text { Require a pulse jet of } \\
\text { high pressure }\end{array}$ & 2.33 \\
\hline Production & $\begin{array}{l}\text { Development, } \\
\text { efficiency, } \\
\text { replacing } \\
\text { equipment and } \\
\text { entrance fees }\end{array}$ & $\begin{array}{l}\checkmark \text { Low explosive } \\
\text { hazard }\end{array}$ & 1.33 & $\begin{array}{l}\checkmark \text { Possibility of reusing } \\
\text { the filter after cleaning } \\
\checkmark \text { High cost raw } \\
\text { material }\end{array}$ & 2.33 \\
\hline HSE risks & $\begin{array}{l}\text { Personnel's } \\
\text { safety, fires, the } \\
\text { damage to the } \\
\text { production } \\
\text { network }\end{array}$ & $\begin{array}{l}\checkmark \text { Minimum risk fire } \\
\text { and explosion }\end{array}$ & 1.66 & $\begin{array}{l}\checkmark \text { Unavailability of } \\
\text { testing technology } \\
\checkmark \text { Environmental } \\
\text { hazards caused by waste } \\
\text { production from } \\
\text { changing period } \\
\checkmark \text { Exposure to particles } \\
\text { and fibers released } \\
\text { during the changing and } \\
\text { maintenance }\end{array}$ & 1.66 \\
\hline \multicolumn{3}{|c|}{$\begin{array}{l}\text { Arithmetic mean and standard deviation of benefit- cost } \\
\text { rating }\end{array}$} & $\begin{aligned} \mathrm{X} & =1.7 \\
\mathrm{SD} & =0.36\end{aligned}$ & $\begin{array}{l}\text { Arithmetic mean and } \\
\text { standard deviation of } \\
\text { cost rating }\end{array}$ & $\begin{array}{c}\mathrm{X}=2 \\
\mathrm{SD}=0.43\end{array}$ \\
\hline
\end{tabular}


Table 4. Results of cost-benefit criteria and elements evaluation for wet ESP

\begin{tabular}{|c|c|c|c|c|c|}
\hline $\begin{array}{l}\text { Cost-benefit } \\
\text { criteria }\end{array}$ & $\begin{array}{l}\text { Elements of cost- } \\
\text { benefit criteria }\end{array}$ & $\begin{array}{c}\text { Benefit elements } \\
\text { examples }\end{array}$ & $\begin{array}{l}\text { Average } \\
\text { benefit } \\
\text { rate }\end{array}$ & Examples of cost elements & $\begin{array}{l}\text { Average } \\
\text { cost rate }\end{array}$ \\
\hline Financial & $\begin{array}{l}\text { Investment, required } \\
\text { equipments to build } \\
\text { and launch }\end{array}$ & $\begin{array}{l}\checkmark \text { Low operating costs } \\
\text { except in the high } \\
\text { efficiency } \\
\checkmark \text { Low operating costs } \\
\checkmark \text { Collectors fewer plate } \\
\text { compared to dry type }\end{array}$ & 2.33 & 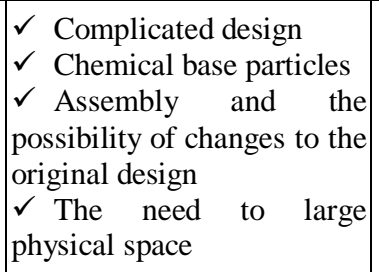 & 1.33 \\
\hline Personnel & \begin{tabular}{|l} 
Training, \\
internships, internal, \\
external period, \\
absence from work
\end{tabular} & $\begin{array}{l}\checkmark \text { Short-term training } \\
\text { courses }\end{array}$ & 2.33 & $\begin{array}{l}\checkmark \text { Training of technicians } \\
\text { for technical changes in the } \\
\text { structure }\end{array}$ & 1.33 \\
\hline $\begin{array}{l}\text { Service and } \\
\text { maintenance }\end{array}$ & \begin{tabular}{|l|} 
\\
Operating period, \\
services, operational \\
energy, maintenance \\
and service
\end{tabular} & $\begin{array}{l}\checkmark \text { Reuse the filter after } \\
\text { cleaning } \\
\checkmark \text { Energy consumption } \\
\text { depends on the size of the } \\
\text { particles that not exist in } \\
\text { other devices } \\
\checkmark \text { Low operating costs } \\
\text { except in the high range }\end{array}$ & 1.66 & $\mid \begin{array}{l}\checkmark \text { Maintenance service } \\
\checkmark \text { Low rate of efficiency } \\
\text { with incorrect design }\end{array}$ & 1.33 \\
\hline $\begin{array}{l}\text { Environmental } \\
\text {-social }\end{array}$ & $\begin{array}{lr}\text { Release of toxic } \\
\text { substances, waste } \\
\text { management, the } \\
\text { rule of law, } \\
\text { efficiency }\end{array}$ & $\begin{array}{l}\checkmark \text { High purification } \\
\text { efficiency } \\
\checkmark \text { ACCEPTANCE acidic } \\
\text { and corrosive gases } \\
\checkmark \text { Ability to collect the } \\
\text { gas and particles at the } \\
\text { same time }\end{array}$ & 2.33 & 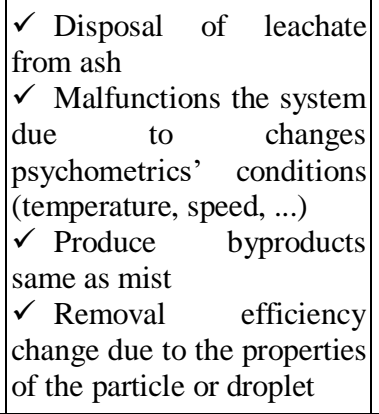 & 1.66 \\
\hline $\begin{array}{l}\text { Customer } \\
\text { orientation }\end{array}$ & $\begin{array}{l}\text { Investment, new } \\
\text { products, the need of } \\
\text { new information }\end{array}$ & $\begin{array}{l}\checkmark \text { This technology is } \\
\text { native } \\
\checkmark \text { Unexpected products in } \\
\text { plaster and cement } \\
\text { factories are part of a high } \\
\text { quality product }\end{array}$ & 2 & $\begin{array}{l}\checkmark \text { Materials do not } \\
\text { separated of the size } \\
\checkmark \text { Corrosion in the pages } \\
\text { due to sludge production }\end{array}$ & 1.33 \\
\hline Production & \begin{tabular}{|l|} 
Development, \\
efficiency, replacing \\
equipment and \\
entrance fees
\end{tabular} & $\begin{array}{l}\checkmark \text { Acceptance of a wide } \\
\text { range of gas and dust high } \\
\text { volume } \\
\checkmark \text { The electromechanical } \\
\text { equipment in the existing }\end{array}$ & 1.66 & 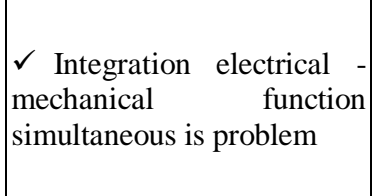 & 1.33 \\
\hline HSE risks & $\begin{array}{l}\text { Personnel's } r \text { safety, } \\
\text { fires, the damage to } \\
\text { the } \\
\text { network }\end{array}$ & $\begin{array}{l}\checkmark \text { Impossibility of release } \\
\text { of captured dust } \\
\checkmark \text { Minimal risk of fire and } \\
\text { explosion }\end{array}$ & 1.66 & $\begin{array}{l}\checkmark \text { Recycling problems } \\
\checkmark \text { The possibility to } \\
\text { respiratory exposure } \\
\checkmark \text { Generated waste and } \\
\text { contamination soil, etc. } \\
\checkmark \text { Generated noise and } \\
\text { vibration } \\
\checkmark \text { The creation of high- } \\
\text { frequency waves }\end{array}$ & 1.33 \\
\hline \multicolumn{3}{|c|}{ Arithmetic mean and standard deviation of benefit- cost rating } & $\begin{array}{c}X=1.42 \\
\mathrm{SD}=0.16\end{array}$ & $\begin{array}{l}\text { Arithmetic mean and } \\
\text { standard deviation of cost } \\
\text { rating }\end{array}$ & $\begin{array}{c}X=1.954 \\
S D=0.4\end{array}$ \\
\hline
\end{tabular}


Table 5. Results of cost-benefit criteria and elements evaluation for ESP bag filter

\begin{tabular}{|c|c|c|c|c|c|}
\hline $\begin{array}{l}\text { Cost-benefit } \\
\text { criteria }\end{array}$ & $\begin{array}{l}\text { Elements of } \\
\text { Cost-benefit } \\
\text { criteria }\end{array}$ & $\begin{array}{l}\text { Benefit elements } \\
\text { examples }\end{array}$ & $\begin{array}{l}\text { Average } \\
\text { benefit } \\
\text { rate }\end{array}$ & $\begin{array}{l}\text { Examples of cost } \\
\text { elements }\end{array}$ & $\begin{array}{l}\text { Average } \\
\text { cost rate }\end{array}$ \\
\hline Financial & \begin{tabular}{|l|} 
Investment, \\
required \\
equipments to \\
build and launch
\end{tabular} & $\begin{array}{l}\checkmark \text { High-efficiency filter } \\
\text { is not necessary } \\
\checkmark \text { Plate cost is less } \\
\text { compared with Wet ESP }\end{array}$ & 2.33 & $\begin{array}{l}\checkmark \text { Power supply } \\
\checkmark \text { High pressure loss } \\
\checkmark \text { ESP plate }\end{array}$ & 2.66 \\
\hline Personnel & $\begin{array}{l}\text { Training, } \\
\text { internships, } \\
\text { internal, external } \\
\text { period, absence } \\
\text { from work }\end{array}$ & $\begin{array}{l}\checkmark \text { Initial training for } \\
\text { commissioning }\end{array}$ & 2 & $\begin{array}{l}\checkmark \text { Training } \\
\text { technicians of } \\
\text { technical changes in } \\
\text { the structure } \\
\checkmark \text { More specialized } \\
\text { training for multiple } \\
\text { functions (mechanical } \\
\text {-electrical) }\end{array}$ & 1.66 \\
\hline $\begin{array}{l}\text { Service and } \\
\text { maintenance }\end{array}$ & $\begin{array}{l}\text { Operating period, } \\
\text { services, } \\
\text { operational } \\
\text { energy, } \\
\text { maintenance and } \\
\text { service }\end{array}$ & $\begin{array}{l}\checkmark \text { Reuse the filter after } \\
\text { cleaning } \\
\checkmark \text { Control at the time } \\
\text { charged and neutral } \\
\text { particles }\end{array}$ & 1.66 & $\begin{array}{l}\checkmark \text { Special } \\
\text { maintenance } \quad-\quad \text { high } \\
\text { cost }\end{array}$ & 2.66 \\
\hline $\begin{array}{l}\text { Environmental } \\
\text {-social }\end{array}$ & $\begin{array}{l}\text { Release of toxic } \\
\text { substances, waste } \\
\text { management, the } \\
\text { rule of law, } \\
\text { efficiency }\end{array}$ & $\begin{array}{l}\checkmark \text { High filtration } \\
\text { efficiency for a wide } \\
\text { range of particles }\end{array}$ & 2 & 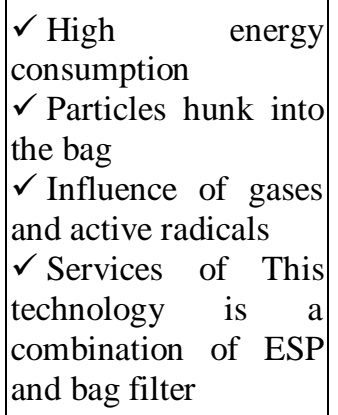 & 2 \\
\hline $\begin{array}{l}\text { Customer } \\
\text { orientation }\end{array}$ & $\begin{array}{|lr|}\text { Investment, } & \text { new } \\
\text { products, the } & \text { need } \\
\text { of } & \text { new } \\
\text { information } & \end{array}$ & $\begin{array}{l}\checkmark \text { This technology is } \\
\text { native } \\
\checkmark \text { A wide range of } \\
\text { particles are extraction } \\
\text { able }\end{array}$ & 2 & $\begin{array}{l}\checkmark \text { Reuse the filter } \\
\text { after cleaning }\end{array}$ & 1.33 \\
\hline Production & $\begin{array}{l}\text { Development, } \\
\text { efficiency, } \\
\text { replacing } \\
\text { equipment and } \\
\text { entrance fees }\end{array}$ & $\begin{array}{l}\checkmark \text { Large systems needed } \\
\text { to the large-scale due to } \\
\text { the production }\end{array}$ & 2 & $\begin{array}{l}\checkmark \text { The creation of } \\
\text { return Corona into the } \\
\text { reactor }\end{array}$ & 2 \\
\hline HSE risks & $\begin{array}{l}\text { Personnel's } \\
\text { safety, fires, the } \\
\text { damage to the } \\
\text { production } \\
\text { network }\end{array}$ & $\begin{array}{l}\checkmark \text { Fire risk is low due to } \\
\text { low load on the bag } \\
\text { filter } \\
\checkmark \text { Bag Filter is the pre- } \\
\text { treatment }\end{array}$ & 2.33 & $\begin{array}{l}\checkmark \text { Waste production } \\
\checkmark \text { The possibility of } \\
\text { electrocution } \\
\checkmark \text { Produce noise and } \\
\text { vibration } \\
\checkmark \text { Need to earth } \\
\text { system }\end{array}$ & 2.33 \\
\hline \multicolumn{3}{|c|}{$\begin{array}{l}\text { Arithmetic mean and standard deviation of benefit- cost } \\
\text { rating }\end{array}$} & $\begin{array}{l}X=2.1 \\
\mathrm{SD}=0.5\end{array}$ & $\begin{array}{l}\text { Arithmetic mean and } \\
\text { standard deviation of } \\
\text { cost rating }\end{array}$ & $\begin{aligned} \mathrm{X} & =2.1 \\
\mathrm{SD} & =0.23\end{aligned}$ \\
\hline
\end{tabular}


Table 6. Results of cost-benefit criteria and elements evaluation for Nano fibers beds

\begin{tabular}{|c|c|c|c|c|c|}
\hline $\begin{array}{l}\text { Cost-benefit } \\
\text { criteria }\end{array}$ & $\begin{array}{l}\text { Elements of } \\
\text { cost-benefit } \\
\text { criteria }\end{array}$ & $\begin{array}{l}\text { Benefit elements } \\
\text { examples }\end{array}$ & $\begin{array}{l}\text { Average } \\
\text { benefit } \\
\text { rate }\end{array}$ & $\begin{array}{l}\text { Examples of cost } \\
\text { elements }\end{array}$ & $\begin{array}{l}\text { Average } \\
\text { cost rate }\end{array}$ \\
\hline Financial & \begin{tabular}{|l|} 
Investment, \\
required \\
equipments to \\
build and launch
\end{tabular} & $\begin{array}{l}\checkmark \text { The use of polymer } \\
\text { salt for making } \\
\text { nanofibers } \\
\checkmark \text { Access to the } \\
\text { production of polymer } \\
\text { composite within }\end{array}$ & 2 & $\begin{array}{l}\checkmark \text { Electrospinning device } \\
\text { is essential for nanofibers } \\
\text { production } \\
\checkmark \text { Power supply with high } \\
\text { voltage }(25 \mathrm{kV})\end{array}$ & 2.33 \\
\hline Personnel & \begin{tabular}{|l|} 
Training, \\
internships, \\
internal, \\
external period, \\
absence rrom \\
work
\end{tabular} & $\begin{array}{l}\checkmark \text { Initial training to } \\
\text { operate machines }\end{array}$ & 2.33 & $\begin{array}{l}\checkmark \text { The experts are allowed } \\
\text { to work with nanofibers } \\
\text { platforms }\end{array}$ & 2.66 \\
\hline $\begin{array}{l}\text { Service and } \\
\text { maintenance }\end{array}$ & $\begin{array}{l}\text { operating } \\
\text { period, } \\
\text { Services, } \\
\text { Operational } \\
\text { Energy, } \\
\text { Maintenance } \\
\text { and Service }\end{array}$ & $\begin{array}{lr}\checkmark \text { Low } & \text { energy } \\
\text { operation } & \\
\checkmark & \text { Simple } \\
\text { function } & \text { mechanical }\end{array}$ & 1.66 & 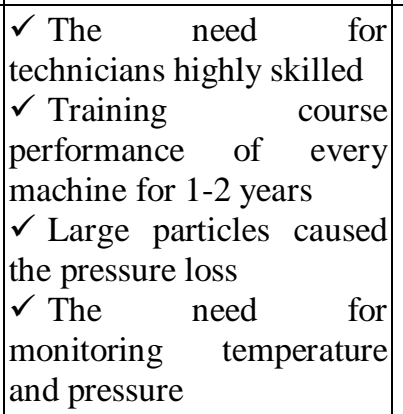 & 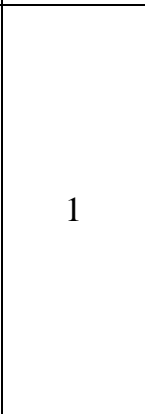 \\
\hline $\begin{array}{l}\text { Environmental } \\
\text {-social }\end{array}$ & \begin{tabular}{|l|} 
Release of toxic \\
substances, \\
Waste \\
Management, \\
the rule of law, \\
efficiency
\end{tabular} & $\begin{array}{l}\checkmark \text { Ability to filtration } \\
\text { simultaneously } \text { of } \\
\text { gases - particles and } \\
\text { acid mist } \\
\checkmark \text { Elimination of odors } \\
\checkmark \text { High quality factor } \\
\text { filter }\end{array}$ & 2 & $\begin{array}{ll}\checkmark & \text { Residual production } \\
\checkmark & \text { High pressure loss } \\
\checkmark & \text { Quality factor fall at } \\
\text { high temperatures }\end{array}$ & 2.33 \\
\hline $\begin{array}{l}\text { Customer } \\
\text { orientation }\end{array}$ & \begin{tabular}{|l|} 
Investment, new \\
products, the \\
need of new \\
information \\
\end{tabular} & \begin{tabular}{|l|}
$\checkmark$ Elimination \\
active radicals organic \\
and inorganic \\
$\checkmark$ Simultaneous \\
elimination of particles \\
and gases \\
\end{tabular} & 2.33 & $\begin{array}{l}\checkmark \text { Reuse the filter after } \\
\text { cleaning }\end{array}$ & 2.33 \\
\hline Production & $\begin{array}{l}\text { Development, } \\
\text { efficiency, } \\
\text { replacing } \\
\text { equipment and } \\
\text { entrance fees }\end{array}$ & \begin{tabular}{|lrr|}
$\checkmark$ Easily & used & for \\
treating & \multicolumn{2}{c}{ gaseous } \\
pollutants & at & the \\
nanoscale & & \\
$\checkmark$ & Composite & polymer \\
\multicolumn{2}{l}{ could be replaced } & with \\
material available & \\
\end{tabular} & 2.33 & $\begin{array}{l}\checkmark \text { Costly } \\
\text { technology }\end{array}$ & 2.33 \\
\hline HSE risks & $\begin{array}{l}\text { Personnel's } \\
\text { safety, fires, the } \\
\text { damage to the } \\
\text { production } \\
\text { network }\end{array}$ & $\begin{array}{ll}\checkmark & \text { Ability to fire } \\
\checkmark & \text { High pressure loss }\end{array}$ & 2 & $\begin{array}{l}\checkmark \text { Skin and respiratory } \\
\text { exposure to nano materials } \\
\checkmark \text { Generated noise and } \\
\text { vibration } \\
\checkmark \text { Replaceable fiber is } \\
\text { waste material }\end{array}$ & 1.66 \\
\hline \multicolumn{3}{|c|}{$\begin{array}{l}\text { Arithmetic mean and standard deviation of benefit- cost } \\
\text { rating }\end{array}$} & $\begin{aligned} \mathrm{X} & =2.1 \\
\mathrm{SD} & =0.57\end{aligned}$ & $\begin{array}{l}\text { Arithmetic mean and } \\
\text { standard deviation of cost } \\
\text { rating }\end{array}$ & $\begin{array}{c}X=2.14 \\
\mathrm{SD}=0.32\end{array}$ \\
\hline
\end{tabular}


Table 7. Average and coefficient of variation in cost-benefit of particle control technologies

\begin{tabular}{c|c|c|c|c|c|c}
\hline $\begin{array}{c}\text { Controlling } \\
\text { technology }\end{array}$ & $\begin{array}{c}\text { Average } \\
\text { benefit rate }\end{array}$ & $\begin{array}{c}\text { Average } \\
\text { cost rate }\end{array}$ & $\begin{array}{c}\text { Benefit coefficient } \\
\text { of variation }\end{array}$ & $\begin{array}{c}\text { Cost coefficient } \\
\text { of variation }\end{array}$ & $\begin{array}{c}\text { Benefit-cost } \\
\text { ratio }\end{array}$ & Rating \\
\hline ULPA filter & $1.7 \pm 0.36$ & $2 \pm 0.43$ & $21 \%$ & $21 \%$ & 0.85 & $*$ \\
Wet ESP & $1.954 \pm 0.4$ & $1.42 \pm 0.16$ & $20 \%$ & $11 \%$ & 1.37 & 1 \\
ESP bag filter & $2.10 \pm 0.5$ & $2.10 \pm 0.23$ & $10 \%$ & $23 \%$ & 1 & 3 \\
Nano fibers beds & $2.14 \pm 0.32$ & $2.1 \pm 0.57$ & $14 \%$ & $27 \%$ & 1.01 & 2 \\
\hline
\end{tabular}

\section{Discussion}

\section{ULPA filter technology}

The results in Table 3 show more costs in the financial, personnel, customer service, and production areas. Possible changes to the original design, heat-resistant compounds, maintenance and service, blocked pores and vulnerability to high temperatures, and recycling are the costs of using this technology. High efficiency, information localization, least risk of fire and explosion, and the re-use of filters after cleaning are the benefits of using this technology. The results of previous research study showed that the filtration efficiency of ULPA filter is over $99.999 \%$ with a particle diameter of 0.12 $0.17-\mu \mathrm{m}$ Along with significant costs in maintenance and operations (Liu et al., 2017).

\section{Wet ESP technology}

According to the results Table 4, the financial, personnel, environmental, social, and customer benefits are a result of lower operating costs, short-term training, low power consumption, high temperature tolerance, and high filtration efficiency stemming from the use of this technology. The simultaneous collection of gas and particle is also an advantage of using this technology. The costs of this technology include those incurred in changes to the original design, the need for more physical space, maintenance and servicing, and corrosion caused by the generated sludge. According to the results, the obtained benefits of this technology outweigh the costs. Brandley (2005) announced that ESPs are a common means of reducing PM emissions from boilers, kilns and engines. Collection efficiencies was various, depending on the size and quality of the ESP, but in some cases have reached to upwards of $99 \%$ levels. The economic impact was found to be $\$ 5,430 /$ tpy (Brandley, 2005).

Wet ESP technology provides with many features and benefits including low operating and maintenance costs, ultra low particulate emissions, compatibility with wet flue gas desulfurization (wet FGD) technology, and an optimized system design for sorbent injection technologies. For manufacturers with existing air pollution control equipment, wet ESP upgrades draw praise from customers who are eliminating carryover and costly maintenance. Improvements to water treatment, gas flow distribution and mist elimination enhance performance, reduce operating costs and protect downstream equipment (http://www.babcock.com/technology/pollutioncontrol/particulate).

\section{ESP bag filter technology}

According to the results in Table 5 the benefits of this technology include the removal of environmental emissions and social benefits resulting from the simultaneous 
control of ESP Bag filter and neutral chargeable particles, as well as high profits from pre-filtering bags. The technology however has high financial and design costs, as well as investment-related costs such as the need for supply source, ES pages, and the need for lightning protection systems. Research and education, especially for service and maintenance, have been allocated a big budget. Localization and the reuse of filters after cleaning are the advantages of using this technology. The combination of electrostatic precipitator (ESP) with bag filter technologies, which can filter smaller particles but fail to work effectively on larger particles, were selected as the proper alternative (closeness coefficient $=0.867$ ) $($ Moridi et al., 2017).

\section{Nano fibers beds technology}

Results in Table 6 show that the removal of particles (10 microns and smaller) in nano-technology fibers platform leads to social consequences and damage control, and is therefore beneficial. Recycling is however costly with this technology. Removal of organic and non-organic radicals, simultaneous extraction of particles and gases, odor elimination, low power operation, and access to raw materials and polymer composites are the advantages of using this technology. On the basis of the results captured in Table 7 and T-test (one-sample-test) with a significance level of 5\%, one can relatively have a realistic judgment in estimating the approximate financial, human, environmental costs.

Bennett (2016) announced that nanofiber coatings Plus filter cartridges are another development from Freudenberg. According to Freudenberg, high initial efficiency can be guaranteed because, unlike conventional filters, these filters do not need pre-coating with dust. The corrugated filter medium ensures constant low resistance, reducing power and compressed air consumption, potentially extending the product lifetime. Nanofiber coated filter cartridges are straight for-ward to handle and require minimal maintenance (Bennett, 2016).

\section{Conclusion}

In this study, the problem of choosing the effectively technical and economic for particulate control technologies, were addressed based on the cost benefit analysis method, which is a reliable scientific method for decision-makers. The methodology developed in this work in term of weighting the seven aspects of cost and benefits (financial, personnel, service and maintenance, environmental-social, customer orientation, production, HSE risks) for selected effectively technical and economic particulate control technologies. Furthermore, decision-makers could use the results obtained via this method to better assess their performance in particulate control. The use of hybrid technologies, including Wet-ESP, has been a top priority for research, but it is feasible to use emission sources with limitations that require extensive research.

\section{REFERENCES}

[1] Bennett, A. (2016): Developments in air \& gas filtration technology. - Filtration + Separation 53: 30-35.

[2] Boardman, A. E., Greenberg, D. H., Vining, A. R., Weimer, D. L. (2017): Cost-Benefit Analysis: Concepts and Practice. - Cambridge University Press, Cambridge, UK. 
[3] Bollen, J., van der Zwaan, B., Brink, C., Eerens, H. (2009): Local air pollution and global climate change: A combined cost-benefit analysis. - Resource and Energy Economics31: $161-81$.

[4] Brandley, M. (2005): Best Available Technology for Air Pollution Control: Analysis Guidance and Case Studies for North America. - Commission for Environmental Cooperation (CEC) of North America, Montréal, QC.

[5] Campbell, H. R., Brown, R. P. C. (2010): Benefit-Cost Analysis: Financial and Economic Appraisal using Spreadsheets. - Cambridge University Press, Cambridge, UK.

[6] Cellini, S. R., Kee, J. E. (2010): Cost-Effectiveness and Cost-Benefit Analysis. Handbook of Practical Program Evaluation 3. Jossey-Bass Wiley, San Francisco, CA.

[7] Chen, X., Zhao, L., Özdemir, M. S., Liang, H. (2018): Mixed strategy to allocate resources with air pollution treatment in China: based on the analytic network process and large-group decision-making method. - Environmental Science and Pollution Research: 1-15.

[8] Gao, J., Yuan, Z., Liu, X., Xia, X., Huang, X., Dong, Z. (2016): Improving air pollution control policy in China A perspective based on cost-benefit analysis. - Science of the Total Environment 543: 307-14.

[9] Huang, C., Moran, A. E., Coxson, P. G., Yang, X., Liu, F., Cao, J., Chen, K., Wang, M., He, J., Goldman, L., Zhao, D. (2017): Potential cardiovascular and total mortality benefits of air pollution control in urban China. - Circulation 136: 1575-84.

[10] Jin, J., Cheon, S., Lee, J., Lee, S. Kim, J. (2018): Economic impact of overseas coal bed methane development project on Korean economy. - Energy \& Environment: 2: 0958305X18762153. https://doi.org/10.1177/0958305X18762153.

[11] Lei, J. A. (2005): Fuzzy Multi-Criteria Decision Analysis for Assessing Technologies of air Pollution Abatement at Coal-Fired Power Plants. - M. S. Thesis Engineering in Environment, University of Regina, Regina, Canada.

[12] Liu, G., Xiao, M., Zhang, X., Gal, C., Chen, X., Liu, L., Clements-Croome, D. (2017): A review of air filtration technologies for sustainable and healthy building ventilation. Sustainable Cities and Society. - 32: 375-396.

[13] Min, S. H., Lim, S. Y., Yoo, S. H. (2017): The environmental benefits of reducing thermal discharge from nuclear power generation. - Energy \& Environmental Science 28: 885-894.

[14] Moridi, P., Atabi, F., Nouri, J., Yarahmadi, R. (2017): Selection of optimized air pollutant filtration technologies for petrochemical industries through multiple-attribute decisionmaking. - Journal of Environmental Management 197: 456-63.

[15] Murty, M. N., Dhavala, K. K., Ghosh, M., Singh, R. (2006): Social Cost-Benefit Analysis of Delhi Metro. - Institute of Economic Growth, Delhi.

[16] Nath, B., Cholakov, G. S. (2009): Pollution Control Technologies. - EOLSS Publications, London.

[17] Qld, D. I. (2011): Project Assurance Framework: Cost Benefit Analysis. Analysis. Queensland Department of Infrastructure and Planning, Queensland.

[18] Quah, E. (2007): Cost-Benefit Analysis. - Routledge, London.

[19] Robert, J., Erson, J. R. (1983): An empirical analysis of economic strategies for controlling air pollution. - Journal of Environmental Economics and Management 10: 112-124.

[20] Salehi, M., Tavakkoli-Moghaddam, R. (2008): Project selection by using a fuzzy TOPSIS technique. - International Journal of Mechanical, Aerospace, Industrial, Mechatronic and Manufacturing Engineering 2: 375-380.

[21] Sen, A. (2000): The discipline of cost-benefit analysis. - The Journal of Legal Studies 29: 931-52.

[22] Spiegel, J., Maystre, L. Y. (1998): Environmental Pollution Control and Prevention. - In: Stellman, J. M. (ed.) Encyclopedia of Occupational Health and Safety (4 ${ }^{\text {th }}$ ed.) International Labour Office, Switzerland, Geneva. 
[23] Svensson, L. E. (2016): A Simple Cost-Benefit Analysis of Using Monetary Policy for Financial Stability Purposes. - In: Blanchard, O. J. et al. (eds.) Progress and Confusion: The State of Macroeconomic Policy. MIT Press, Cambridge.

[24] Treasury, Q. (2006): Cost-Benefit Analysis Guidelines Achieving Value for Money in Public Infrastructure and Service Delivery. - Project Assurance Framework Supplementary Guidance Material, Brisbane, Australia.

[25] Vanoye, A. Y., Mendoza, A. (2014): Application of direct regularization techniques and bounded-variable least squares for inverse modeling of an urban emissions inventory. Environmental Science and Pollution Research International 5: 219-25.

[26] Vlachokostas, C. h., Achillas, C. h., Moussiopoulon, N., Banias, G. (2011): Multicriteria Methodological approach to manage urban air pollution. - Atmospheric Environment 41: 4160-4169.

[27] Voorhees, A. S., Sakai, R., Araki, S. h., Sato, H., Otsu, A. (2001): Cost-benefit analysis methods for assessing air pollution control program in urban environments. Environmental Health and Preventive Medicine 6: 63-73.

[28] Yarahmadi, R., Sadoughi, S. H. (2012): Evaluating and prioritizing of performance indices of environment using fuzzy TOPSIS. - Indian Journal of Science and Technology 5: 2713-2719.

[29] Yarahmadi, R., Mortazavi, S. B., Omidkha, M. R., Asilian, H., Khavanin, A., Moridi, P. (2010): Examination of optimized conditions for the conversion of NOx pollution DBD Plasma reactor. - Iranian Journal of Chemistry and Chemical Engineering 29: 133-140.

[30] Yarahmadi, R., Mortazavi, S. B., Moridi, P. (2012): Development of Air Treatment Technology Using Plasma Method. - International Journal of Occupational Hygiene 4: 27-35.

[31] Zeng, X. T., Tong, Y. F., Cui, L., Kong, X. M., Sheng, Y. N., Chen, L., Li, Y. P. (2017): Population-production-pollution nexus based air pollution management model for alleviating the atmospheric crisis in Beijing, China. - Journal of Environmental Management 197: 507-521.

[32] Zhang, Q., Qiu, M., Lai, K., Zhong, N. (2015): Cough and environmental air pollution in China. - Journal of Pulmonary Pharmacology and Therapeutics 35: 132-136.

[33] Zhao, X., Yu, X., Wang, Y., Fan, C. (2016): Economic evaluation of health losses from air pollution in Beijing, China. - Environmental Science and Pollution Research 23: 11716-11728. 